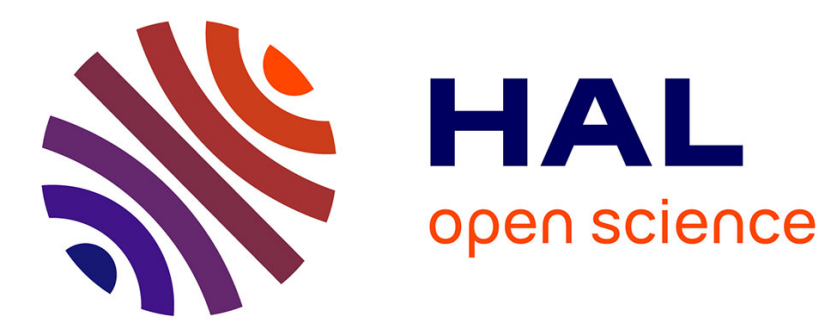

\title{
Coupled optimization of macroscopic structures and lattice infill
}

Perle Geoffroy-Donders, Grégoire Allaire, Georgios Michailidis, Olivier Pantz

\section{To cite this version:}

Perle Geoffroy-Donders, Grégoire Allaire, Georgios Michailidis, Olivier Pantz. Coupled optimization of macroscopic structures and lattice infill. 2020. hal-02429166

\section{HAL Id: hal-02429166 https://hal.science/hal-02429166}

Preprint submitted on 6 Jan 2020

HAL is a multi-disciplinary open access archive for the deposit and dissemination of scientific research documents, whether they are published or not. The documents may come from teaching and research institutions in France or abroad, or from public or private research centers.
L'archive ouverte pluridisciplinaire HAL, est destinée au dépôt et à la diffusion de documents scientifiques de niveau recherche, publiés ou non, émanant des établissements d'enseignement et de recherche français ou étrangers, des laboratoires publics ou privés. 


\title{
Coupled optimization of macroscopic structures and lattice infill
}

\author{
Perle Geoffroy-Donders* $\quad$ Grégoire Allaire ${ }^{\dagger}$ \\ Georgios Michailidis ${ }^{\ddagger} \quad$ Olivier Pantz ${ }^{\S}$
}

November 8, 2019

\begin{abstract}
This paper is concerned with the coupled optimization of the external boundary of a structure and its infill made of some graded lattice material. The lattice material is made of a periodic cell, macroscopically modulated and oriented. The external boundary may be coated by a layer of pure material with a fixed prescribed thickness. The infill is optimized by the homogenization method while the macroscopic shape is geometrically optimized by the Hadamard method of shape sensitivity. A first original feature of the proposed approach is that the infill material follows the displacement on the exterior boundary during the geometric optimization step. A second key feature is the dehomogenization or projection step which build a smoothly varying lattice infill from the optimal homogenized properties. Several numerical examples illustrate the effectiveness of our approach in 2-d, which is especially convenient when considering design-dependent loads.
\end{abstract}

\section{Introduction}

During the last decades, topology optimization has been well-established in diverse industrial sectors as an efficient approach for conceptual design [9]. The capability to obtain optimized designs without spending significant time in preprocessing attracted the interest of engineers and designers that search to improve their products, especially when the mechanical framework limits the role of intuition and experience (dynamics, acoustics, electro-magnetics, fluids, etc.). The progressive incorporation of manufacturing constraints $[24,25,27,43]$ in available commercial software contributed in reducing the gap between topology optimization results and end-parts.

*PIMM, Paris, FRANCE - perle.geoffroy@polytechnique.edu

${ }^{\dagger}$ CMAP, École Polytechnique, Palaiseau, FRANCE - gregoire.allaire@polytechnique.fr

$¥$ ANSYS Inc. - georgios.michailidis@ansys.com

$\S$ Université Côte d'Azur, LJAD, FRANCE - pantz@unice.fr 
The recent burst of advances in additive manufacturing techniques has a significant impact on the area of topology optimization. The unprecedented design freedom endowed by such techniques provides the capability to realize designs of extreme geometric complexity. Moreover, the ability to print features at a very small scale, has revitalized the interest of the homogenization method for design optimization $[10,36,7,1,37]$ and of various multi-scale design frameworks, as in [34]. Infill structures are typical examples of multi-scale design: the boundary of the shape is a macroscopic feature, while the interior of the shape is not homogeneous but filled by some architectured material at a smaller meso-scale. The infill material can typically have any geometry: periodic or non-regular, homogeneous or graded, etc. [33]. The interest in infill designs originates from diverse perspectives. First, it constitutes an additional design freedom, hence enlarges the set of admissible shapes and thus may improve the performance in terms of mass or mechanical efficiency. In addition, infill designs may be chosen for implicit reasons, as they are known to feature a better behavior than homogeneous materials for mechanical problems that are difficult to be included in the optimization problem (buckling, acoustics, etc.) [14, 42].

There already exist several works on the topology optimization of infill structures. However, only few works deal with the concurrent optimization of the shape and the infill part. In [40] Wu et al. presented a density approach with multiple filters and projection schemes in order to optimize coated structures with non-regular infill. In [23] Gröen et al. used the same methodology for coated structures, combined with the homogenization method for the optimization of an orthotropic infill part. After the optimal design has been obtained, a dehomogenization step is applied in order to obtain a classical "black-andwhite" shape. A similar approach, although using different techniques, has been presented in [41].

In this work, we present another approach, based on the coupling of the level-set method [5, 38] and the homogenization method [1]. An infill structure is described by two ingredients: its macroscopic shape and a lattice material filling the interior of the shape. Note that the shape boundary may be coated by a layer of pure material (without holes) having a fixed prescribed thickness. The structure of this lattice material is parametrized by a few variables, which may vary inside the macroscopic shape. The topology optimization process consists in alternating the homogenization method and the level-set method at each iteration, until convergence. With the first one, the parameters of the lattice material are updated, while the macroscopic shape is kept fixed. With the second one, the Hadamard method of shape sensitivity yields a velocity field which concurrently deforms the macroscopic shape and the infill material. The fact that the lattice geometry follows the shape boundary in its displacement during the optimization process is a key feature of our algorithm and is new to the best of our knowledge.

By coupling them, we take advantage of both methods. On the one hand, as was recently shown in several papers [31, 22, 4], the homogenization method is a very efficient method for optimizing a lattice structure, but with no genuine information of the external border. It is efficient because the homogenized 
properties of the lattice are optimized (which only requires a coarse mesh) and in a final dehomogenization step a graded lattice is projected on a possibly very fine mesh. On the other hand, the level-set method is very well suited for the optimization of macroscopic shapes, but it cannot handle lattice structures since it would require a too fine mesh to capture details at the meso-scale of the lattice. A crucial ingredient in our coupling strategy is that the infill density must be bounded from below by a fixed positive threshold. Otherwise, there would be no optimal shape boundary since holes could be achieved by zero density infill. Numerically, the optimization process pushes the shape boundary outside of the computational domain, at the price of introducing very low density infill material in the regions which were exterior to the previous shape. Finally, after the coupled optimization of the shape boundary and of the homogenized infill properties, a post-processing step, called dehomogenization or projection, is performed to recover a modulated and oriented lattice material inside the structure. This step is based on our previous works [4, 21] (see also [31, 23]) and is very economical in terms of CPU time.

Thanks to our proposed approach, infill structures can easily and effectively be optimized under new geometric constraints, without any additional steps. Indeed, contrary to density-based methods (either SIMP or homogenization), the genuine external border of the current structure is known here. We focus in the present work on three kind of problems. The first one is the coupled optimization of a macroscopic structure and its infill. Since there is no control of the thickness of the boundary of the structure in this first approach, a second case is considered, which consists in optimizing a structure, with its infill, featuring additionally a coating with given thickness. Eventually, the third problem is the optimization of an infill structure under design-dependent load, namely hydrostatic pressure here.

Section 2 is concerned with the setting of the problem. For simplicity, we focus on compliance minimization in 2-d (our approach can be extended to other objective functions and to 3-d without major conceptual difficulties but at the price of several technicalities that are not discussed here). We recall our definition of lattice materials as periodic structures, macroscopically modulated and oriented as in [4]. In Section 3, the various derivatives of the objective function and constraints are computed with respect either to the shape or to the lattice parameters (size and orientation). The resulting shape derivative is different from the classical one, used in the level-set method, since the lattice infill is following the shape and therefore contributes an additional term in the shape derivative (see Lemma 3.2). Section 4 describes the algorithm and its numerical implementation. The effectiveness of the method is illustrated by several 2-d numerical examples in Section 5.

\section{Description of the problem}

For the sake of clarity, the present work is restricted to the 2-d case. Nevertheless, all results can be extended to the 3 - $\mathrm{d}$ case (this is well documented for the 
homogenization and the level-set method ; the dehomogenization method has recently been extended to 3 -d in [21]). Let $D \subset \mathbb{R}^{2}$ be the bounded working domain. The admissible shapes or structures, $\Omega \subset D$, are subsets of $D$ which are bounded open sets occupied by an elastic material (infill). Instead of being homogeneous, the material is assumed to feature a micro-structure and is characterized by its homogenized elastic tensor $A^{*}$. The boundary of $\Omega$ is comprised of three disjoint parts, such that $\partial \Omega=\Gamma_{D} \cup \Gamma_{N} \cup \Gamma$, with Dirichlet conditions applied on $\Gamma_{D}$, non-homogeneous Neumann conditions on $\Gamma_{N}$, while $\Gamma$ is traction free. For simplicity, only $\Gamma$ is subject to optimization. The displacement field $u$ is the solution of the elasticity system:

$$
\left\{\begin{array}{ll}
\operatorname{div}\left(A^{*} e(u)\right)=0 & \text { in } \Omega \\
u=0 & \text { on } \Gamma_{D} \\
A^{*} e(u) n=g & \text { on } \Gamma_{N} \\
A^{*} e(u) n=0 & \text { on } \Gamma
\end{array},\right.
$$

where $e(u)=\frac{1}{2}\left(\nabla u+\nabla u^{T}\right)$ is the strain tensor and $g$ denotes the boundary forces.

\section{$2.1 \quad$ Lattice infill}

Among the plethora of materials that can be used as infill, lattices are of particular interest since they are easily parametrized and manufactured. In addition, structural optimization including lattices has been well studied $[32,11]$ and recent works on the dehomogenization of optimized lattice infills [31, 22, 4] provide a useful framework for their direct exploitation in additive manufacturing.

As lattice we consider a graded (spatially varying) elastic composite material made of two phases: void and an isotropic solid phase whose elastic tensor will be denoted by $A$. The homogenized tensor of the composite material $A^{*}$ is constructed in two steps. First, we consider a square periodicity cell, denoted $Y_{0}(m)$, with a rectangular hole, parametrized via two geometric parameters $m=$ $\left(m_{1}, m_{2}\right) \in[0 ; 1]^{2}$ (see Figure 1). Using (modulated) periodic homogenization $[37,1]$, we obtain an homogenized orthotropic elastic tensor, which we denote by $A_{0}^{*}(m)$. Then, we orient $A_{0}^{*}(m)$ according to an angle $\alpha \in L^{\infty}(\Omega,[0 ; 2 \pi])$. Hence the complete elasticity tensor reads:

$$
A^{*}(m(x), \alpha(x))=R(\alpha(x)) A_{0}^{*}(m(x)) R(\alpha(x))^{T},
$$

where $R(\alpha(x))$ is the fourth-order tensor corresponding to a rotation of angle $\alpha(x)$ for symmetric matrices. To simplify the notation, we omit in the sequel the spatial dependency of $A^{*}$.

The material density is denoted $\rho(m) \in[0 ; 1]$ and its value is

$$
\rho(m)=1-m_{1} m_{2} \text {. }
$$

When $\rho(m)=0$ (which happens only for $m_{1}=m_{2}=1$ ), the composite material is void, while $\rho(m)=1$ (which happens either for $m_{1}=0$ or for $m_{2}=0$ ) corresponds to a fully solid material (with a thin crack if one of the $m_{i}$ is not zero). 


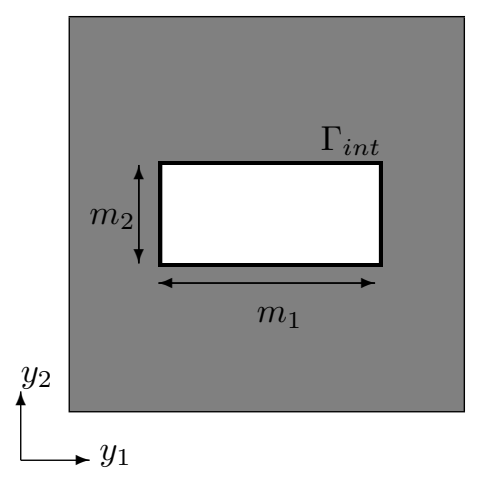

Figure 1: Periodicity cell $Y_{0}(m)$.

Remark 1. We emphasize that our approach is not restricted to the particular micro-structure of Figure 1 and can be easily generalized to any other parametrized orthotropic lattice material.

\subsection{Optimization problem}

\subsubsection{General setting}

The goal is to optimize the shape $\Omega$, as well as the parameters of its graded lattice infill $(m, \alpha)$, in order to minimize a cost function denoted by $J(\Omega, m, \alpha)$. We restrict ourselves to the compliance of the structure, namely:

$$
J(\Omega, m, \alpha)=\int_{\Omega} A^{*}(m, \alpha) e(u): e(u) d x,
$$

where $u$ is solution of (1). As usual, a mass constraint is imposed on the structure. The minimization problem reads:

$$
\begin{array}{ll}
\inf _{\Omega \in \mathcal{U}_{a d}, m \in \mathcal{P}_{a d}, \alpha \in L^{\infty}(\Omega,[0 ; 2 \pi])} & \int_{\Omega} A^{*}(m, \alpha) e(u): e(u) d x \\
\text { such that } & M(\Omega, m)=\int_{\Omega} \rho(m) d x=M_{T},
\end{array}
$$

where $\rho(m)$ is defined by $(2), M_{T}$ denotes the target mass, $\mathcal{U}_{a d}$ the set of admissible shapes

$$
\mathcal{U}_{a d}=\left\{\Omega \subset D \quad \mid \quad \Gamma_{N} \subset \partial \Omega, \Gamma_{D} \subset \partial \Omega\right\},
$$

and $\mathcal{P}_{a d}$ the set of admissible geometric parameters of the lattice :

$$
\mathcal{P}_{a d}=L^{\infty}\left(\Omega,[0 ; 1]^{2}\right)
$$




\subsubsection{Constrained setting}

In practice, during the optimization process, in the absence of other constraints, the density of the lattice infill has to be restricted by a minimum value. Indeed, if not, the lattice infill can be void at some places, see Section 5.1.1. In such a case, there is no need to have an exterior boundary and the shape $\Omega$ can simply be the whole domain $D$. To avoid this problem and to get a clearly defined exterior boundary it is therefore required to have a strictly positive minimal density of the infill. It turns out to be much simpler to impose a lower bound on the geometric parameters $m_{i}$ of the lattice, rather than on the density $\rho(m)$. Hence, an upper bound for the size of the rectangular hole is introduced, $0<m_{\max }<1$, and the set of admissible geometric parameters is defined by

$$
\mathcal{P}_{a d}=L^{\infty}\left(\Omega,\left[0 ; m_{\max }\right]^{2}\right) .
$$

The above definition of the constrained set $\mathcal{P}_{a d}$ is not completely satisfactory since it forbids the rectangular hole to cut the cell and thus the microstructure $Y_{0}(m)$ can never be that of a rank-one laminate, made of only two parallel bars (although it is known that rank-one laminates are optimal for uni-axial stress).

Remark 2. If one insists to work only with the density, and not with the parameters $m_{i}$, another possibility is to add to the objective function a penalization term of the type

$$
\int_{\Omega} \mid f\left(\left.\rho(x)\right|^{2} d x,\right.
$$

where $f$ is a penalization function, nearly equal to 1 for low densities and equal to zero for other densities. For a threshold value $0<\rho_{\min }<1$, one example is

$$
f(\rho)= \begin{cases}\frac{1}{2}\left(\cos \left(\frac{\pi \rho}{\rho_{\min }}\right)+1\right) & \text { if } \rho<\rho_{\min }, \\ 0 & \text { if } \rho \geq \rho_{\min } .\end{cases}
$$

This idea has not been pursued further here.

\subsubsection{Coating}

Using a coating for infill structures can be motivated by manufacturing or even functional reasons. In the first case, the coating acts as a protection layer from its environment (dust, external objects, etc.), while the latter holds true when the boundary is of key importance for its mechanical efficiency (acoustics, fluids, electro-magnetics, etc.). Moreover, when a dehomogenization step follows the optimization of a composite material, the post-treatment from useless tiny features proves to be significantly simplified for coated structures.

The coating region can be easily provided by a geometric description using the signed distance function to the domain $\Omega$. 
Definition 2.1. Let $\Omega \subset \mathbb{R}^{2}$ be a Lipschitz open set. The signed distance function to $\Omega$, denoted by $d_{\Omega}: \mathbb{R}^{2} \rightarrow \mathbb{R}$, is defined as:

$$
d_{\Omega}(x)=\left\{\begin{array}{ll}
-d(x, \partial \Omega) & \text { if } x \in \Omega \\
0 & \text { if } x \in \partial \Omega \\
d(x, \partial \Omega) & \text { if } x \notin \bar{\Omega}
\end{array},\right.
$$

where $d(\cdot, \partial \Omega)$ is the usual Euclidean distance to the boundary $\partial \Omega$.

Then, the coating region of uniform size $h$, denoted $\Omega_{h}$ in the following, is defined by:

$$
\Omega_{h}=\left\{x \in \Omega \quad \mid \quad d_{\Omega}(x)>-h\right\} .
$$

Imposing numerically the coating area around the boundary of the evolving shape can be done in several ways. Here, for simplicity we choose a penalization approach (see Remark 3 for another possible multi-material approach). To enforce the coating region, a penalization function is introduced which forces the material density $\rho(m)$, defined by $(2)$, to be close to 1 :

$$
P_{\text {coat }}(\Omega, m)=\left(\int_{\Omega_{h}}(\rho(m)-1) d x\right)^{2} .
$$

Then, a penalized optimization problem is solved:

$$
\begin{array}{cl}
\inf _{\Omega \in \mathcal{U}_{a d}, m \in \mathcal{P}_{a d}, \alpha \in L^{\infty}(\Omega,[0 ; 2 \pi])} & \int_{\Omega} A^{*}(m, \alpha) e(u): e(u) d x+\gamma P_{\text {coat }}(\Omega, m) \\
\text { s.t. } & M(\Omega, m)=\int_{\Omega} \rho(m) d x=M_{T}
\end{array}
$$

for a given parameter $\gamma>0$. When $\gamma$ is set to a large enough value, the penalization $P_{\text {coat }}$ is vanishingly small, meaning that the coating region $\Omega_{h}$ is filled with the pure material.

Remark 3. Another, more explicit, way of enforcing the coating amounts to modify the elasticity tensor, so that it takes the coating region into consideration. Assuming that the coating is made of the same phase $A$ as in the composite, the total elastic tensor $\hat{A}^{*}$ reads:

$$
\hat{A}^{*}=\chi_{\Omega_{h}} A+\left(1-\chi_{\Omega_{h}}\right) A^{*}(m, \alpha),
$$

where $\chi_{\Omega_{h}}$ denotes the characteristic function of the domain $\Omega_{h}$. As a result, the optimization becomes a multi-material problem, which introduces several difficulties with respect to the shape derivation (see [3] for a detailed presentation on multi-material shape and topology optimization). To avoid these technicalities and since in numerical practice the penalization approach works well we did not pursue the multi-material approach. 


\section{Sensitivity analysis}

This section establishes first order derivatives of the involved functionals with respect to the optimization parameters, in order to use them in a gradient optimization algorithm.

\subsection{Derivation with respect to the lattice parameters}

As is well known, optimizing the compliance (3) for the state equation (1) is a self-adjoint problem [2]. In other words, no adjoint state is required. Its derivative with respect to the parameters of the micro-structure, (see [1] for example), reads:

$$
\frac{\partial J}{\partial m_{i}}(\Omega, m, \alpha)=-\int_{\Omega} \frac{\partial A^{*}}{\partial m_{i}}(m, \alpha) e(u): e(u) d x,
$$

where

$$
\frac{\partial A^{*}}{\partial m_{i}}(m, \alpha)=R(\alpha) \frac{\partial A_{0}^{*}}{\partial m_{i}}(m) R(\alpha)^{T} .
$$

Since there is no explicit formula for the homogenized tensor $A_{0}^{*}(m)$ with respect to its parameters $m_{i}$, the computation of $\frac{\partial A_{0}^{*}}{\partial m_{i}}(m)$ is performed numerically (see [4] for details). A similar formula holds true for the derivative of the compliance with respect to the orientation angle $\alpha$, i.e.

$$
\frac{\partial J}{\partial \alpha}(\Omega, m, \alpha)=-\int_{\Omega} \frac{\partial A^{*}}{\partial \alpha}(m, \alpha) e(u): e(u) d x .
$$

Although formula (9) could be computed, in practice we do not use it, since for compliance minimization the optimal orientation of orthotropic materials is explicitly given by [32] and coincides with the principal directions of the stress field $\sigma=A^{*}(m, \alpha) e(u)$. By choosing to align the vector $(\cos (\alpha) ; \sin (\alpha))$ with the eigenvector of $\sigma$ of smallest eigenvalue (possibly negative), the angle $\alpha$ is then defined modulo $\pi$.

\subsection{Shape differentiation}

\subsubsection{Definition}

We rely on Hadamard's method to compute shape derivatives and more specifically we adopt the approach of Murat and Simon [35]. Let $\Omega$ be a smooth reference open set, $\theta \in W^{1, \infty}\left(\mathbb{R}^{2}, \mathbb{R}^{2}\right)$ and Id the identity map. If $\theta$ is small enough, the map $\mathrm{Id}+\theta$ is a diffeomorphism in $\mathbb{R}^{2}$. Hence we can consider the domains:

$$
\Omega_{\theta}=(\operatorname{Id}+\theta)(\Omega) .
$$


Definition 3.1. A functional $F(\Omega)$ is said to be shape differentiable at $\Omega$ if there exists a continuous linear form $F^{\prime}(\Omega)$ on $W^{1, \infty}\left(\mathbb{R}^{2}, \mathbb{R}^{2}\right)$ such that, for all $\theta \in W^{1, \infty}\left(\mathbb{R}^{2}, \mathbb{R}^{2}\right):$

$$
F((I+\theta)(\Omega))=F(\Omega)+F^{\prime}(\Omega)(\theta)+o(\theta) \quad \text { with } \lim _{\theta \rightarrow 0} \frac{|o(\theta)|}{\|\theta\|}=0,
$$

where $\|\theta\|$ is the norm of $\theta$ in $W^{1, \infty}\left(\mathbb{R}^{2}, \mathbb{R}^{2}\right)$ and $F^{\prime}$ is the shape derivative of the functional $F$.

We recall a classical result on the shape derivative of volume integrals (see [2] for a proof).

Lemma 3.1. For $f \in W^{1,1}\left(\mathbb{R}^{2}\right)$ define

$$
F(\Omega)=\int_{\Omega} f(x) d x .
$$

Then $F$ is differentiable at $\Omega$ and, for all $\theta \in W^{1, \infty}\left(\mathbb{R}^{2} ; \mathbb{R}^{2}\right)$,

$$
F^{\prime}(\Omega)(\theta)=\int_{\Omega} \operatorname{div}(\theta(x) f(x)) d x=\int_{\partial \Omega} \theta(s) \cdot n(s) f(s) d s .
$$

\subsubsection{Compliance}

To compute the shape derivative of the compliance (3), one has first to define how the material properties, i.e. the elastic tensor of the graded lattice infill, depend on shape variations. As already said, there are two modeling possibilities.

In the first case, the graded material is defined (or extended somehow) in the whole domain $D$ and is kept fixed when the macroscopic shape $\Omega$ is deformed by $\operatorname{Id}+\theta$. The new shape $(\operatorname{Id}+\theta) \Omega$ is filled with the same infill, defined on $D \cap(\operatorname{Id}+\theta) \Omega$, see Figure 2(a). In such a case, the standard framework described in [6] applies and the classical results presented therein could be used.

In the second case, the infill $A^{*}(x)$ is also transported by the diffeomorphism $\operatorname{Id}+\theta$, see Figure 2(b). Hence the new shape $\Omega_{\theta}=(\operatorname{Id}+\theta) \Omega$ is filled with the transported infill material, whose tensor is given by:

$$
A_{\theta}^{*}=A^{*}(m, \alpha) \circ(\mathrm{Id}+\theta)^{-1} .
$$

We found this second choice more sound both from a physical and numerical perspective and adopted it for our implementation. In particular, it implies that the lattice infill follows the deformation of the shape $\Omega$. The interpretation of (10) is that, in a Lagrangian setting, i.e., mapping back the infill to the reference configuration, the tensor $A_{\theta}^{*} \circ(\operatorname{Id}+\theta)=A^{*}(m, \alpha)$ is independent of the vector field $\theta$.

Lemma 3.2. Assume the infill parameters $(m, \alpha)$ are $C^{1}$ on $\Omega$ and the solution $u$ of (1) belongs to $H^{2}(\Omega)^{2}$ (this is true for a smooth shape and a smooth 

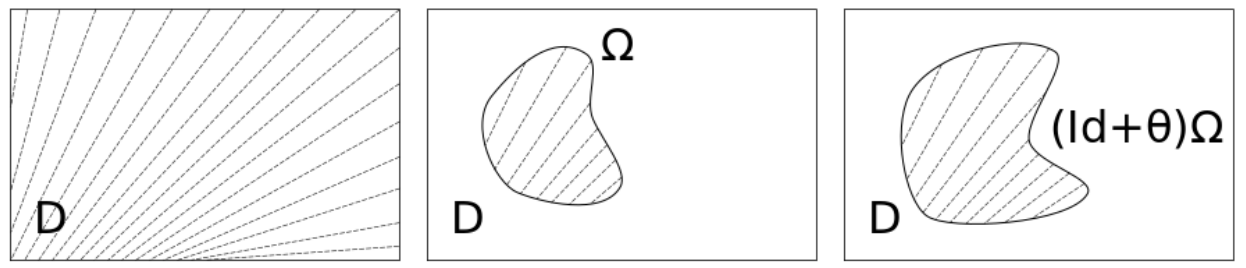

(a) The lattice material is defined on the working domain $D$ (left). The shape $\Omega$ is filled by this lattice material (center). When the shape is deformed (right), the lattice material is kept fixed, defined in $D$.
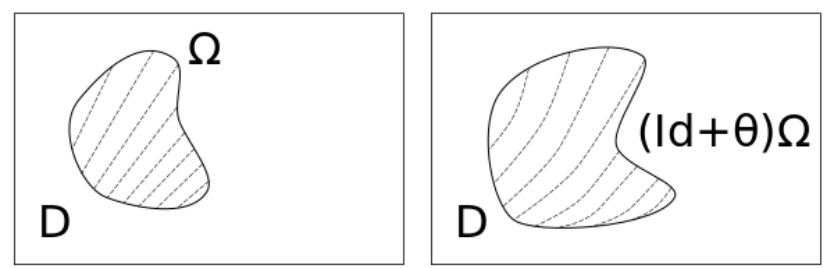

(b) The lattice material is defined only on the shape $\Omega$ (left). When the shape $\Omega$ is deformed by ( $\operatorname{Id}+\theta)$, the lattice material is also transported by $(\mathrm{Id}+\theta)$ (right).

Figure 2: Two possible variations of the shape $\Omega$ and its infill by a diffeomorphism $(\mathrm{Id}+\theta)$. The dotted lines may represent the isolines of the local density of the lattice material. 
loading). For any $\theta \in W^{1, \infty}\left(\mathbb{R}^{2}, \mathbb{R}^{2}\right)$ such that $\theta=0$ on $\Gamma_{D} \cup \Gamma_{N}$, the shape derivative of the compliance, defined by (3), reads:

$J^{\prime}(\Omega, m, \alpha)(\theta)=-\int_{\Gamma} A^{*}(m, \alpha) e(u): e(u) \theta \cdot n d s+\int_{\Omega} \theta \cdot \nabla A^{*}(m, \alpha) e(u): e(u) d x$.

Remark 4. In (11) the notation $J^{\prime}$ denotes the shape derivative, computed for given $m$ and $\alpha$. It is thus a partial derivative.

Proof. We rely on the Lagrangian method of Céa (see e.g. [2]). For vector fields $\theta \in W^{1, \infty}\left(\mathbb{R}^{2}, \mathbb{R}^{2}\right)$, we consider deformed sets $\Omega_{\theta}=(\operatorname{Id}+\theta) \Omega$ with infill $A_{\theta}^{*}$, defined by (10), and introduce the following Lagrangian

$$
\mathcal{L}(\theta, v, q)=\int_{\Gamma_{N}} g \cdot v d s-\int_{\Omega_{\theta}} A_{\theta}^{*} e(v): e(q) d x+\int_{\Gamma_{N}} g \cdot q d s,
$$

where $v$ and $q$ belong to $H^{1}(\Omega)^{2}$ and vanish on $\Gamma_{D}$. As usual, the Lagrangian is defined as the sum of the objective function (the compliance, defined by (3), is also equal to the work done by the load). Since it is assumed that $\theta=0$ on $\Gamma_{D} \cup \Gamma_{N}$, i.e. that these boundaries are kept fixed, the three variables $(\theta, v, q)$ are independent, which ensures that the following computations make sense.

Differentiating the Lagrangian with respect to $q$ and requiring that this partial derivative vanishes yields the variational formulation for $v=u$, solution of (1). Differentiating the Lagrangian with respect to $v$ and requiring that this partial derivative vanishes yields the variational formulation for the adjoint which is nothing else than $q=u$, solution of (1). Finally, differentiating the Lagrangian with respect to $\theta$ and evaluating it at $(v, q)=(u, u)$ gives the shape derivative of the objective function (see [1], [2])

$$
\begin{aligned}
\frac{\partial \mathcal{L}}{\partial \theta}(0, v, q)(\theta)=J^{\prime}(\Omega, m, \alpha)(\theta)= & -\int_{\Gamma} A^{*}(m, \alpha) e(u): e(u) \theta \cdot n d s \\
& +\int_{\Omega} \theta \cdot \nabla A^{*}(m, \alpha) e(u): e(u) d x .
\end{aligned}
$$

by applying Lemma 3.1 and computing the derivative of $\theta \rightarrow A_{\theta}^{*}$ by a simple Taylor expansion for small $\theta$

$$
A_{\theta}^{*}=A^{*}(m, \alpha) \circ(\mathrm{Id}+\theta)^{-1}=A^{*}(m, \alpha)-\theta \cdot \nabla A^{*}(m, \alpha)+o(\theta) .
$$

Developing a bit further the second term of formula (11) for the shape deriva- 
tive of the compliance, we obtain:

$$
\begin{aligned}
& J^{\prime}(\Omega, m, \alpha)(\theta)= \\
& -\int_{\Gamma} R(\alpha) A_{0}^{*}(m) R(\alpha)^{T} e(u): e(u) \theta \cdot n d s \\
& +\int_{\Omega} R(\alpha) \frac{\partial A_{0}^{*}}{\partial m}(m) R(\alpha)^{T} e(u): e(u) \theta \cdot \nabla m d x \\
& +\int_{\Omega}\left(\frac{\partial R}{\partial \alpha}(\alpha) A_{0}^{*}(m) R(\alpha)^{T}+R(\alpha) A_{0}^{*}(m) \frac{\partial R^{T}}{\partial \alpha}(\alpha)\right) e(u): e(u) \theta \cdot \nabla \alpha d x .
\end{aligned}
$$

As mentioned in section 3.1, since the optimization with respect to the angle orientation is performed explicitly at each iteration using Pedersen formulas [32], the last term in (13) vanishes because of the optimality conditions with respect to the orientation angle $\alpha$. Therefore, denoting by $\alpha^{*}$ the optimal orientation, the shape derivative of the compliance reduces to:

$$
\begin{aligned}
& J^{\prime}\left(\Omega, m, \alpha^{*}\right)(\theta)= \\
& -\int_{\Gamma} R\left(\alpha^{*}\right) A_{0}^{*}(m) R\left(\alpha^{*}\right)^{T} e(u): e(u) \theta \cdot n d s \\
& +\int_{\Omega} R\left(\alpha^{*}\right) \frac{\partial A_{0}^{*}}{\partial m}(m) R\left(\alpha^{*}\right)^{T} e(u): e(u) \theta \cdot \nabla m d x .
\end{aligned}
$$

Formula (14) is used in our numerical simulations.

\subsubsection{Mass}

Following the same analysis as in subsection 3.2.2, the shape derivative of the mass $M(\Omega, m)$ reads:

$$
M^{\prime}(\Omega, m)(\theta)=\int_{\Gamma} \rho(m) \theta . n d s-\int_{\Omega} \frac{\partial \rho}{\partial m}(m) \theta \cdot \nabla m d x
$$

\subsubsection{Coating functional}

The coating functional $P_{\text {coat }}$ is posed on the coating domain $\Omega_{h}$, which is defined in (6) via the signed distance function to the domain $\Omega$. Therefore, the shape derivative of the signed distance $d_{\Omega}$ is required. Details concerning the shape derivation of $d_{\Omega}$ can be found in $[17,18,3]$. Only the basic information that we need for our results are recalled here.

Definition 3.2. The skeleton (or ridge) of $\Omega$, denoted by $\Sigma$, is the set of points $x \in \mathbb{R}^{2}$ such that the minimum in

$$
d(x, \partial \Omega)=\min _{y \in \partial \Omega}|x-y|
$$

is achieved by at least two distinct points $y$ of $\partial \Omega$. 
Proposition 3.3. Assume $\Omega$ is an open set of class $C^{1}$ and fix a point $x \notin \Sigma$, outside the skeleton of $\partial \Omega$. The map $\theta \mapsto d_{(\mathrm{Id}+\theta) \Omega}(x)$ is Gateaux differentiable at $\theta=0$, as an application from $W^{1, \infty}\left(\mathbb{R}^{2}, \mathbb{R}^{2}\right)$ into $\mathbb{R}$ and its derivative is

$$
d_{\Omega}^{\prime}(\theta)(x)=-\theta\left(p_{\partial \Omega}(x)\right) \cdot n\left(p_{\partial \Omega}(x)\right),
$$

where $p_{\partial \Omega}(x)$ is the orthogonal projection of $x$ on $\partial \Omega$, which is uniquenely defined for $x \notin \Sigma$.

Our main result, which generalizes Lemma 3.1, is the following.

Proposition 3.4. For $f \in W^{1,1}\left(\mathbb{R}^{2}\right)$ define

$$
K(\Omega)=\int_{\Omega_{h}} f(x) d x .
$$

For a smooth open set $\Omega$ of class $C^{2}$ and a sufficiently small thickness $h>0$, its shape derivative reads, for any $\theta \in W^{1, \infty}\left(\mathbb{R}^{2}, \mathbb{R}^{2}\right)$,

$$
K^{\prime}(\Omega)(\theta)=\int_{\partial \Omega}(f(x)-(1-h H) f(x-h n(x))) \theta(x) \cdot n(x) d s,
$$

where $H=\operatorname{div} n$ is the mean curvature of $\partial \Omega$.

Proof. Let $\zeta$ be the function defined from $\mathbb{R}$ to $\mathbb{R}$ by

$$
\zeta(t)=\left\{\begin{array}{cc}
1 & \text { if }-h<t<0 \\
0 & \text { otherwise }
\end{array}\right.
$$

Then, by definition (6), one has

$$
K(\Omega)=\int_{\mathbb{R}^{2}} \zeta\left(d_{\Omega}(x)\right) f(x) d x .
$$

If $\Omega$ is $C^{2}$ and $h>0$ is small enough, the skeleton does not touch or cross $\Omega_{h}$ and one can differentiate the signed distance function everywhere in $\Omega_{h}$. Therefore, using proposition 3.3, the shape derivative of $K(\Omega)$ is:

$$
\begin{aligned}
K^{\prime}(\Omega)(\theta) & =\int_{\mathbb{R}^{2}} f(x) \zeta^{\prime}\left(d_{\Omega}(x)\right) d_{\Omega}^{\prime}(\theta)(x) d x \\
& =-\int_{\mathbb{R}^{2}} f(x) \zeta^{\prime}\left(d_{\Omega}(x)\right) \theta\left(p_{\partial \Omega}(x)\right) \cdot n\left(p_{\partial \Omega}(x)\right) d x \\
& =\int_{\partial \Omega} f(x) \theta(x) \cdot n(x) d s-\int_{\Gamma_{h}} f(x) \theta\left(p_{\partial \Omega}(x)\right) \cdot n\left(p_{\partial \Omega}(x)\right) d s
\end{aligned}
$$

because $\zeta^{\prime}(t)=\delta(t+h)-\delta(t)$ where $\delta$ is the Dirac mass at 0 , and $\Gamma_{h}$ is the inner boundary of the coating $\Omega_{h}$. Then, recalling that, for $x \in \Omega_{h}, p_{\partial \Omega}(x)=$ $x-d_{\Omega}(x) n(x)$, and performing the change of variable $\tilde{x}=p_{\partial \Omega}(x)$ in the integral on $\Gamma_{h}$, which has the Jacobian $(1-h H)>0$, one gets

$$
\int_{\Gamma_{h}} f(x) \theta\left(p_{\partial \Omega}(x)\right) \cdot n\left(p_{\partial \Omega}(x)\right) d s=\int_{\partial \Omega} f(\tilde{x}-h n(\tilde{x})) \theta(\tilde{x}) \cdot n(\tilde{x}) d s,
$$


from which the desired result follows. In truth, to make this computation fully rigorous, one has first to regularize the step function $\zeta$ with a small parameter $\epsilon>0$ and then pass to the limit in the derivative as $\epsilon$ goes to zero. This is a standard process that we safely leave to the reader.

The above result leads straightforwardly to the derivative of the coating penalization term $P_{\text {coat }}(\Omega, m)$ where, as before, the infill is assumed to follow the shape deformation.

Proposition 3.5. For a smooth open set $\Omega$ of class $C^{2}$ and a sufficiently small thickness $h>0$, the map $\theta \rightarrow P_{\text {coat }}\left((\operatorname{Id}+\theta) \Omega, m \circ(\operatorname{Id}+\theta)^{-1}\right)$ is Gateaux differentiable at $\theta=0$, as an application from $W^{1, \infty}\left(\mathbb{R}^{2}, \mathbb{R}^{2}\right)$ into $\mathbb{R}$, and its derivative reads:

$$
\begin{aligned}
P_{\text {coat }}^{\prime}(\Omega, m)(\theta)= & 2\left(\int_{\Omega_{h}}(\rho(m)-1) d x\right)\left(-\int_{\Omega_{h}} \frac{\partial \rho}{\partial m}(m) \nabla m \cdot \theta d x\right. \\
& \left.+\int_{\Gamma}(\rho(m(s))-(1-h H) \rho(m(s-h n(s)))) \theta(s) \cdot n(s) d s\right) .
\end{aligned}
$$

\section{Numerical implementation}

\subsection{Level-set description}

From a numerical point of view the shape $\Omega$ is represented on a fixed mesh of the working domain $D$ by the level-set method [30]. A level-set function $\phi$ is introduced and the shape $\Omega \subset D$ is described implicitly by

$$
\begin{cases}\phi(x)=0 & \text { if } x \in \partial \Omega \cap D \\ \phi(x)<0 & \text { if } x \in \Omega \\ \phi(x)>0 & \text { if } x \in D \backslash \bar{\Omega}\end{cases}
$$

Usually, the deformation of the shape $\Omega$ is performed by solving a non-linear Hamilton-Jacobi advection equation where the scalar normal velocity is given by the shape derivative. However, in the present context, the shape derivative in (14) or (17) is made of two terms: one classical term on the boundary, depending only on the normal component of the vector field $\theta$, and another new term inside the shape, depending on all components of $\theta$. Therefore, from the very structure of the shape derivative, it is clear that the advection velocity must be vectorvalued, and not merely a scalar normal component. Therefore, one cannot use the usual non-linear Hamilton-Jacobi advection equation. Rather, denoting by $\theta$ the velocity field deduced from shape differentiation (see the next subsection to find out how it is precisely obtained), we use the following linear transport equation

$$
\frac{\partial \phi}{\partial t}(t, x)+\theta(x) \cdot \nabla \phi(t, x)=0 \text { in }[0, d t] \times D,
$$


where $t$ is some pseudo-time variable and the final time $d t$ corresponds to the descent step in the optimization ( $d \mathrm{t}$ has nothing to do with the time step for the numerical resolution of (18)). Note that the velocity field $\theta$ is used for both advection of the shape and of the infill properties. Numerically, we use the advect library [12] to solve (18) on an unstructured mesh. Moreover, the descent step $d t$ is adapted as follows. At each iteration, if the newly computed homogenized structure is accepted, it is increased by $20 \%$. On the contrary, if the newly computed structure is rejected, it is divided by two. The initialization of $d t$ is given by:

$$
d t=\frac{h_{\max }}{\|\theta\|_{L^{\infty}(D)}}
$$

where $h_{\max }$ is the maximal size of the edges of the mesh. The idea is to prevent the shape to move too much between two iterations. In order to avoid significant distortions of the level-set function, leading to numerical errors, as well as to define accurately the coating area, the level-set function $\phi(x)$ is re-initialized at each iteration as the signed-distance function of the current $\Omega$, using the mshdist library [15]. Finally, the classical "ersatz" material approach [6] is used to extend the state equation (1) in the whole working domain $D$.

\subsection{Velocity extension and regularization}

As shown in section 3.2, the shape derivative for functionals including "transported" material properties is no more a surface integral, as in the classical framework of Hadamard's method, where only the normal component of the advection field on the shape boundary is of interest. Instead, the vector field $\theta$ is defined both on the boundary and inside the shape $\Omega$. Furthermore, to solve the transport equation (18), the advection field must be defined on the whole working domain $D$, thus $\theta$ needs to be extended to $D \backslash \Omega$.

We now explain how to deduce a vector field $\theta$ from the shape derivatives (14) or (17), which is defined everywhere in $D$ and is indeed a descent direction. According to Hadamard theory, $\theta$ should belong to the Lipschitz space $W^{1, \infty}\left(D, \mathbb{R}^{2}\right)$ but, as is common numerical practice, we content ourselves in defining $\theta$ in the (slightly less smooth) Sobolev space $H^{1}\left(D, \mathbb{R}^{2}\right)$. In any case, a typical shape derivative is of the type

$$
J^{\prime}(\Omega, m)(\theta)=\int_{\partial \Omega} j(x) \theta \cdot n d s+\int_{\Omega} k(x) \theta \cdot \nabla m d x
$$

where $j$ and $k$ are two given scalar integrands. A naive choice would amount to choose

$$
\theta(x)= \begin{cases}-j(x) n & \text { on } \partial \Omega \\ -k(x) \nabla m(x) & \text { in } \Omega\end{cases}
$$

which is indeed a descent direction for the objective function $J(\Omega, m)$ but has the main disadvantage of being discontinuous at the boundary $\partial \Omega$. Therefore, another, more educated, choice of $\theta$ has to be made. Following the classical 
idea of derivative regularization [6], [16], [13], we identify the directional derivative with the $H^{1}\left(D, \mathbb{R}^{2}\right)$ scalar product. More precisely, an advection velocity field $\theta$ is computed as the solution of the following variational formulation in $H^{1}\left(D, \mathbb{R}^{2}\right)$ : for any test function $\xi \in H^{1}\left(D, \mathbb{R}^{2}\right)$,

$$
\int_{D}\left(\eta^{2} \nabla \theta: \nabla \xi+\theta \cdot \xi\right) d x=-J^{\prime}(\Omega, m, \alpha)(\xi),
$$

where $J^{\prime}$ is the shape derivative of the functional $J$, e.g. (14), and $\eta>0$ is a small regularization coefficient (typically of the order of a mesh cell size). Taking $\xi=\theta$ in (19) ensures that indeed the solution $\theta$ is a descent direction for $J(\Omega, m, \alpha)$. Furthermore, $\theta$ is well defined in the entire working domain $D$ and not only on $\Omega$.

To compute the surface integral that appears in the right-hand side of (19) on a fixed mesh, we use a volumic approximation as proposed in [29]. The boundary integral of a function $\psi$ defined on $D$ is numerically approximated as:

$$
\int_{\partial \Omega} \psi d s \approx \int_{D} \psi(x) \delta_{\epsilon}(x) d x
$$

where

$$
\delta_{\epsilon}(x)= \begin{cases}0 & \text { if }\left|d_{\Omega}(x)\right|>\epsilon, \\ \frac{1}{2 \epsilon}\left(1+\cos \left(\frac{\pi}{\epsilon} d_{\Omega}(x)\right)\right) & \text { otherwise, }\end{cases}
$$

and $\epsilon>0$ is a small coefficient of the order of the mesh size.

\subsection{Optimization algorithm}

We combine several optimization methods: augmented Lagrangian for shape optimization, gradient descent with projection for the unit-cell parameters and explicit optimization for the orientation angle.

\subsubsection{Augmented Lagrangian}

In this section we describe a simple augmented Lagrangian algorithm for minimizing a cost function $J(\Omega)$ under equality constraints

$$
P_{i}(\Omega)=0, \quad i=1, \ldots, I .
$$

Of course, there are other more efficient optimization algorithms in topology optimization that have been successfully combined with the level-set method $[20,39]$, but it is not the purpose of the present paper to discuss the important issue of optimization efficiency. Following [28] we introduce the augmented Lagrangian function:

$$
L(\Omega, \ell, \mu)=J(\Omega)-\sum_{i=1}^{I} \ell_{i} P_{i}(\Omega)+\sum_{i=1}^{I} \frac{\mu_{i}}{2} P_{i}^{2}(\Omega),
$$


where $J(\Omega)$ is the cost function, $\ell=\left(\ell_{i}\right)_{i=1, \ldots, I}$ the Lagrange multipliers and $\mu=\left(\mu_{i}\right)_{i=1, \ldots, I}$ the penalty parameters, used to enforce the constraints at convergence. The Lagrange multipliers are updated at each iteration $n$ according to the relation $\ell_{i}^{n+1}=\ell_{i}^{n}-\mu_{i} P_{i}\left(\Omega_{n}\right)$ (see [28] for more details). We also increase the penalty parameters every 5 iterations.

The augmented Lagrangian is used here only for the mass constraint. It is not used for the coating constraint of Subsection 2.2.3 since, in practice, a classical simple penalization is very efficient and yields quickly a dense coating, as desired.

\subsubsection{Pseudocode}

To optimize the objective function $J(\Omega, m, \alpha)$ we rely on an alternate direction algorithm. More precisely, the shape $\Omega$ is updated by the level-set method every $n_{0}$ iterations, while the lattice infill parameters $(m, \alpha)$ are updated at all other iterations, that is $\left(n_{0}-1\right)$ times in a row. In practice, we set $n_{0}=5$. The algorithm is stopped when the objective function is no longer significantly decreasing, namely $J_{n}-J_{n+1}<10^{-8}$, where $J_{n}$ is the value of the objective function at iteration $n$ (which is is of order 1 in all our examples). Moreover, a maximal amount of iterations is imposed, in practice here, 200 iterations.

The algorithm reads as follows:

1. Initialization of the shape $\Omega_{0}$ and of the lattice parameters $\left(m_{0}, \alpha_{0}\right)$.

2. Iteration for $n \geq 0$ :

(a) Computation of the solution $u_{n}$ of the linearized elasticity system (1).

(b) If $n \neq 0$ modulo $n_{0}$, update the lattice infill parameters:

i. Computation of the descent direction, using (8).

ii. Updating the geometric parameters $m_{n+1}$ by a projected gradient step.

iii. Updating the orientation $\alpha_{n+1}$ by using Pedersen formulas.

iv. Verification that the new infill improves the objective function, else, reduction of the step size and rejection of the new infill.

v. The shape is kept: $\Omega_{n+1}=\Omega_{n}$.

(c) If $n=0 \bmod n_{0}$, udpate the macroscopic shape:

i. Computation of the descent direction $\theta_{n+1}$

ii. Updating the shape $\Omega_{n+1}$ and the geometric parameters $m_{n+1}$ by advection.

iii. Updating the orientation $\alpha_{n+1}$ of the lattice material using Pedesern formulas.

iv. Verification that the new shape and the new infill improve the objective function, else, reduction of the time interval $d t$ and rejection of the new shape and of the new infill. 
For details on the projected gradient algorithm for the geometric parameter $m$ and on the Pedersen formula for the orientation $\alpha$, the reader is referred to [4].

\subsection{Dehomogenization process}

The optimized microstructure is not a genuine structure. Indeed, the infill lattice is a homogenized material: it is equivalent to periodic cells of infinitely small size. In other words, the optimized homogenized structure is not straightforwardly manufacturable. Hence, it has first to be dehomogenized before it can be built. The dehomogenization process was first introduced by Pantz and Trabelsi [31], for rank laminates materials. It has been further generalized to other microstructures, in 2-d [4, 22] and in 3-d [21]. Here, the same method as in [4] is used and we content ourselves in simply recalling the main ideas below.

The dehomogenization consists in building a sequence of genuine structures, that converges to the optimized homogenized one. Let $\psi(y, m)$ be a level-set function describing the perforated unit periodic cell $Y_{0}(m)$ (this level-set function $\psi$ has nothing to do with the other level-set function $\phi$ which defines the shape $\Omega$ ). In other words, $y=\left(y_{1}, y_{2}\right) \rightarrow \psi(y, m)$ is a periodic function, with period $[0 ; 1]^{2}$, such that

$$
Y_{0}(m)=\left\{y \in[0 ; 1]^{2} \quad \mid \quad \psi(y, m)<0\right\} .
$$

If the orientation of the periodic cells is kept constant in the whole working domain $D$, namely $\alpha(x)=0$, an obvious reconstruction amounts to mesh the working domain with squares of size $\varepsilon$ and to project in each square the corresponding local microstructure, parametrized by the local values of the geometric parameters $m$. The corresponding non-oriented structure $\Omega_{\varepsilon}^{n o}$ is given by:

$$
\Omega_{\varepsilon}^{n o}=\left\{x \in \Omega \quad \mid \psi\left(\frac{x}{\varepsilon}, m(x)\right)<0\right\} .
$$

If the cell orientation $\alpha$ varies in the working domain, the above idea has to be modified. A naive idea would be to simply rotate each cell by $\alpha$, without deforming it. However, it cannot work since two neighbouring squares with different orientation may not be well connected to each other: either they overlap, or there is a gap between them. In order to take into account the local orientation and to yield well connected genuine structures, cells have to be slightly distorted, see Figure 3. This distortion is mathematically given by a vector field, $\varphi: \mathbb{R}^{2} \rightarrow \mathbb{R}^{2}$, a diffeomorphism whose inverse maps the periodic square grid, Figure 3(a), on a distorted grid where each cell is optimally oriented, Figure $3(\mathrm{c})$. (The notation $\varphi$ for this diffeomorphism should not be confused with $\phi$ for the level-set function of $\Omega$.) The grid map $\varphi(x)$ is deduced from the optimal angle $\alpha(x)$. Indeed its gradient has to be locally aligned with the axes of the cell, whose directions are given by $\alpha$, namely

$$
\nabla \varphi(x)=e^{r(x)}\left(\begin{array}{cc}
\cos \alpha(x) & \sin \alpha(x) \\
-\sin \alpha(x) & \cos \alpha(x)
\end{array}\right)
$$




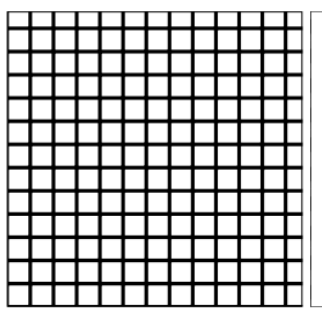

(a) Regular grid

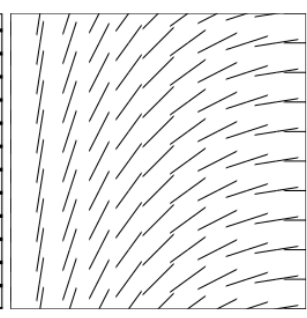

(b) Direction field

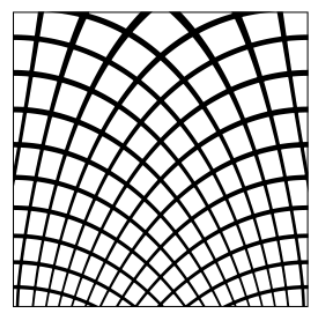

(c) Distorted grid

Figure 3: A regular grid (a) is associated to a direction field (b), giving the local orientation of each cell: it yields a distorted grid (c)

where $r(x)$ is a scalar dilatation field. To solve the above equation for $\varphi$ and $r$, the orientation angle $\alpha$ has to satisfy a conformality condition, namely it must be harmonic. For theoretical, as well as practical details, on the solution process for $\varphi$, the reader is referred to [4].

Eventually, a sequence of genuine, or projected, structures $\Omega_{\varepsilon}(\varphi, m)$, taking into account the orientation of the cells is simply given by composing the levelset function $\psi$, the grid map $\varphi$ and the parameter field $m$ as follows:

$$
\Omega_{\varepsilon}(\varphi, m)=\left\{x \in \Omega \quad \mid \psi\left(\frac{\varphi(x)}{\varepsilon}, m(x)\right)<0\right\} .
$$

The shape $\Omega_{\varepsilon}(\varphi, m)$ is a modulated and oriented periodic domain (recall that $y \rightarrow \psi(y, m)$ is periodic). When $\varepsilon$ is going to zero, $\Omega_{\varepsilon}$ is converging to the optimized homogenized structure characterized by the homogenized tensor $A^{*}(m, \alpha)$.

\section{$5 \quad$ Numerical examples}

Our topology optimization method is implemented in the finite element software FreeFem $++[26]$ (see [8] for the use of FreeFem ++ in optimal design). All unknowns are discretized using $P_{1}$-functions. 


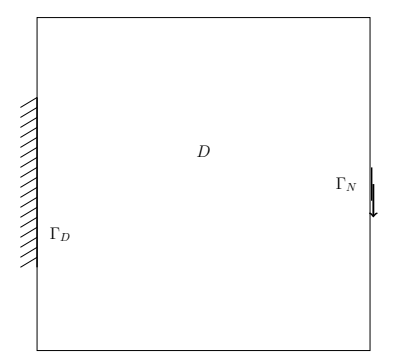

Figure 4: Boundary conditions for the cantilever test case

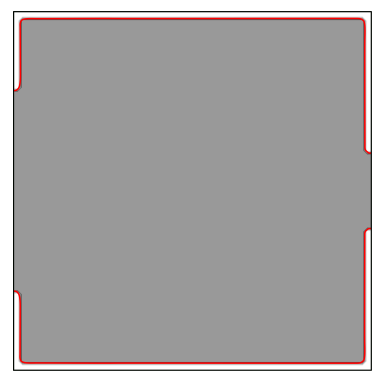

Figure 5: Density $\rho=0.5$ of the initialization structure for the cantilever test case (the boundary of $\Omega$ is the red line)

\subsection{Non-coated structure}

We first discuss numerical results of the optimization process for structures, without any coating.

\subsubsection{Cantilever case}

Our optimization algorithm is tested with a cantilever, see Figure 4 for the boundary conditions. The domain size is $20 \times 20$ and it is discretized by a structured triangular mesh, featuring about 800 vertices. A unit vertical load is applied on a central segment of length 1 on the right side. The structure is clamped on a central segment of length 10 on the left side. The target mass is fixed to $30 \%$ of the working domain, i.e. $M_{T}=120$. The initial shape $\Omega$ is displayed on Figure 5: it is slightly smaller than the whole working domain $D$ and its border contains the Dirichlet and the Neumann boundaries, respectively $\Gamma_{D}$ and $\Gamma_{N}$. Its lattice infill is homogeneous and $m_{1}=m_{2}=\sqrt{0.5} \approx 0.7$, yielding an homogeneous density $\rho=0.5$.

The optimized densities and shapes $\Omega$ for three values of the threshold $m_{\max }$, 1, 0.9 and 0.8 (see Subsection 2.2.2), are displayed on Figure 6. The final values of the objective function, the compliance and the mass are given on Table 1. For these three designs, the mass constraint is satisfied $\left(M_{T}=120\right)$. The compliance and the area of the shape $\Omega$, which is equal to $\int_{\Omega} d x$ and is different from the weight $\int_{\Omega} \rho(m) d x$, are given for comparison. The higher the maximal length 


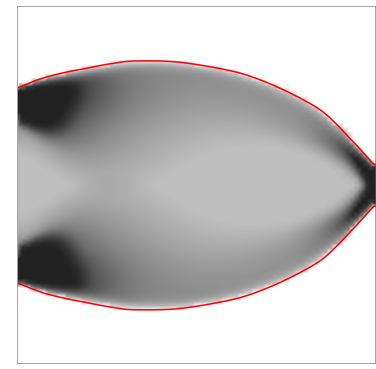

(a) $m_{\max }=0.8$

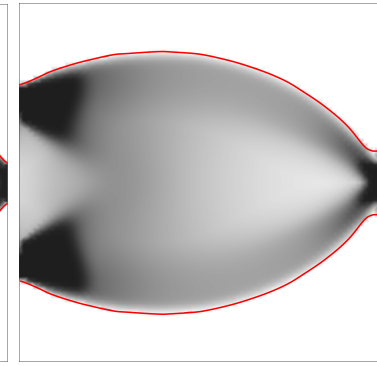

(b) $m_{\max }=0.9$

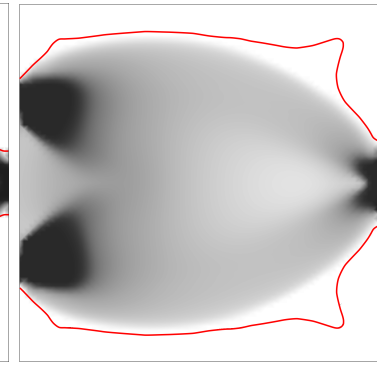

(c) $m_{\max }=1.0$

Figure 6: Cantilever test case: optimized density for different values of $m_{\max }$. The boundary of the shape $\Omega$ is the red line.

of the hole $m_{\max }$, the higher the area of $\Omega$. Indeed, as previously mentioned in Section 2.2.2, when the infill can have a zero density (meaning it is like void), the level-set function, which separates the infill from the external void phase is mechanically meaningless. For $m_{\max }=1$, the level-set do not disappear totally although it could have done so ; nevertheless, the area of $\Omega$ is $85 \%$ of the one of the working domain $D$. However, the external boundary $\partial \Omega$ is surrounded by void (it lies in a white, or void, region on Figure 6(c)) and so has no impact on the final design. The areas of $\Omega$ for $m_{\max }$ equal to 0.9 and 0.8 are smaller. The external boundary $\partial \Omega$ of the design is much more visible on Figures 6 (a) and $6(\mathrm{~b})$, when $m_{\max }=0.9$ or 0.8 , since the transition between the infill and the exterior void phase is sharper.

Besides, the higher $m_{\max }$, the smaller the compliance. Indeed, the set of admissible structures is enriched when $m_{\max }$ increases. Hence the optimized structure can only be more efficient for larger $m_{\max }$. From a mechanical point of view, it means that smooth transitions between the infill and the void (corresponding to large values of $\left.m_{\max }\right)$ are more efficient. However, in practice, after dehomogenization, such transition areas are not manufacturable because they involve very thin bars, that are impossible to manufacture and thus have to be removed before building the structures. This post-process alters the performance of the genuine structures. On the contrary, using a value of $m_{\max }$ smaller than 1 during the optimization step, the optimized structure features no transition phase between infill and void, and thus no very thin bars. No post-process of the genuine structures is then required.

\begin{tabular}{|l|c|c|c|}
\hline$m_{\max }$ & Compliance & Mass & Percentage of area of $\Omega$ w.r.t. $D$ \\
\hline 0.8 & 2.87 & $30.1 \%$ & $57 \%$ \\
0.9 & 2.74 & $29.9 \%$ & $63 \%$ \\
1.0 & 2.63 & $30.2 \%$ & $81 \%$ \\
\hline
\end{tabular}

Table 1: Numerical values for the three optimal designs of Figure 6 


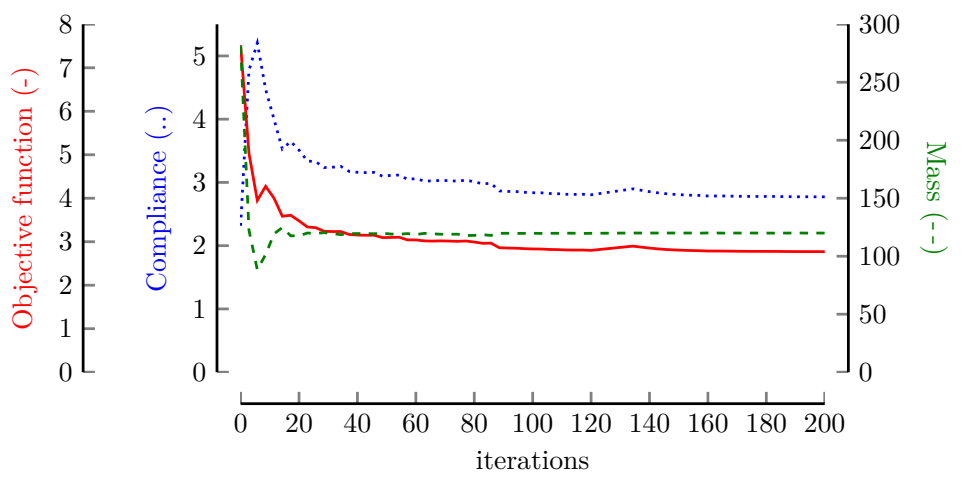

Figure 7: Convergence history of the objective function (red continuous line), the compliance (blue dotted line) and the mass of the structure (green dashed line) for the cantilever with $m_{\max }=0.8$

The convergence history for $m_{\max }=0.8$ is displayed on Figure 7. First, in order to satisfy the mass constraint, the mass decreases abruptly, at the expense of the compliance. After around twenty iterations, the mass constraint is quite respected. The mass does not vary significantly anymore and the compliance decreases gradually.

The optimized homogenized structure for $m_{\max }=0.8$ is dehomogenized in order to yield genuine structures, using the dehomogenization process described in Subsection 4.4 (following [4]). A diffeomorphism $\varphi$ corresponding to the orientation is computed. Then, a sequence of genuine shapes $\Omega_{\varepsilon}(\varphi, m)$ is constructed, see Figure 8 , where $\varepsilon$ stands for the characteristic period of the cells. The smaller $\varepsilon$, the finer the lattice infill.

\subsubsection{Bridge case}

Our approach is now applied to a bridge problem, see Figure 9 for the boundary conditions. A unit vertical load is applied on a central unit segment of the bottom side. The structure is sliding on two unit segments of the bottom border, distant of one unit from the corners. The domain size is $22 \times 13$ and it is discretized by a structured triangular mesh, featuring about 1000 vertices. The target mass is fixed to $30 \%$ of the working domain and $m_{\max }$ is set to 0.8 . The optimized density and the optimized shape $\Omega$ are displayed on Figure 10. A sequence of genuine shapes $\Omega_{\varepsilon}(\varphi, m)$ obtained by the dehomogenization process is displayed in Figure 11.

\subsubsection{Discussion}

Thanks to the shape $\Omega$, tracked by the level-set function $\phi$, there is a clear border between the lattice infill inside the macroscopic structure and the outside of the structure. Consequently, the dehomogenized structures $\Omega_{\varepsilon}(\varphi, m)$ are clean in the sense that no small, thin or disconnected bars appear close to the boundary. 


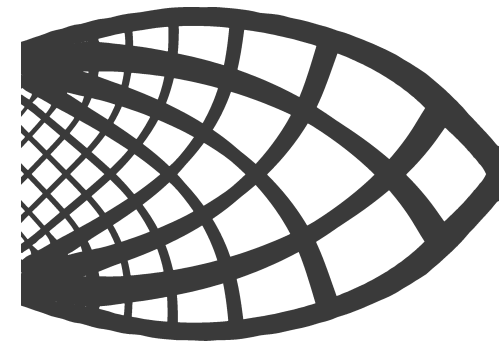

(a) $\varepsilon=0.4$

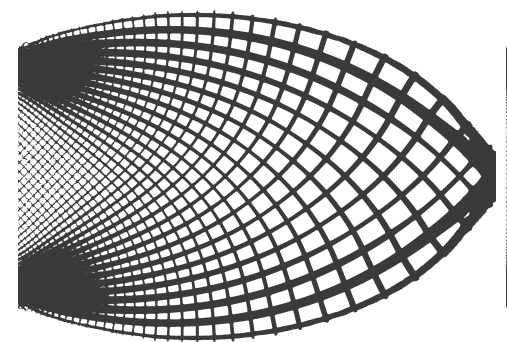

(c) $\varepsilon=0.1$

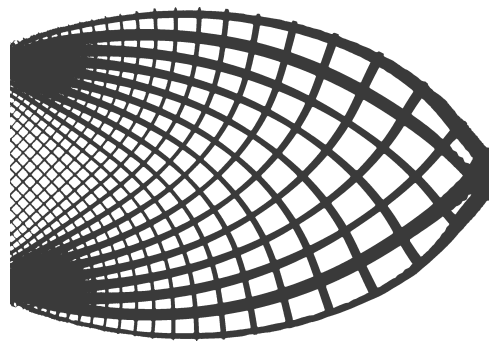

(b) $\varepsilon=0.2$

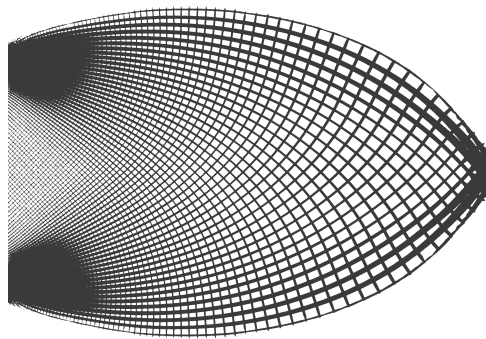

(d) $\varepsilon=0.05$

Figure 8: Projected structures $\Omega_{\varepsilon}(\varphi, m)$ for several $\varepsilon$ in the case of the cantilever, with $m_{\max }=0.8$

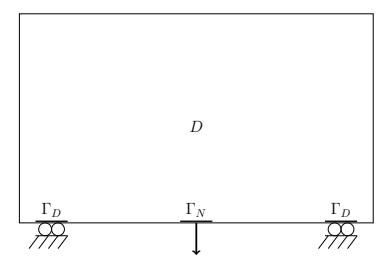

Figure 9: Boundary conditions for a bridge

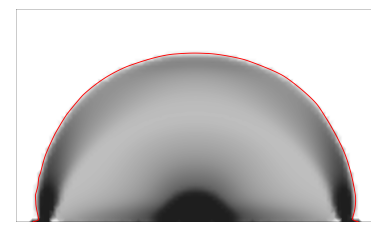

Figure 10: Bridge test case: optimized density for $m_{\max }=0.8$. The boundary of the shape $\Omega$ is the red line. 


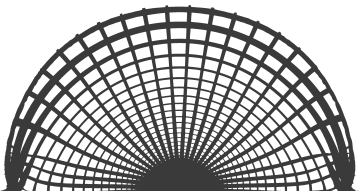

(a) $\varepsilon=0.2$

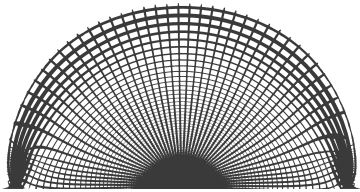

(b) $\varepsilon=0.1$

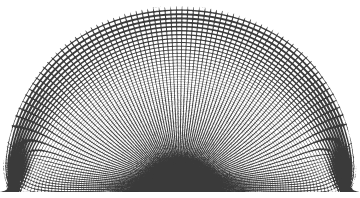

(c) $\varepsilon=0.05$

Figure 11: Projected structures $\Omega_{\varepsilon}(\varphi, m)$ for several $\varepsilon$ in the case of the bridge

This is a great advantage of the coupled optimization method over the topology optimization done only with the homogenization method as in [4]. Indeed, when lattice structures are optimized only with homogenization method, they feature a transition area in the domain $D$, where the density of the lattice goes slowly to zero. This yields very thin bars in this part of the domain during dehomogenization process and a post-treatment is then required to clean the dehomogenized structures. No such post-treatment is necessary here.

Moreover, since the compliance is minimized here, the cells are aligned with the principal directions of the stress, and so with the external boundary defined by the level-set function. Nevertheless, the external border is not perfectly smooth: it features stumps of bars or tiny holes. These small defaults are due to the inherent numerical approximations during the computation of the diffeomorphism $\varphi$. The same post-treatment method, as the one presented in [4], could be used here to clean the structures $\Omega_{\varepsilon}(\varphi, m)$. However we can also fix this problem during the optimization process by imposing a coating to the structure, as we now explain.

\subsection{Coated structures}

Our algorithm for coated structures is tested for a cantilever. The boundary conditions are identical to the ones in the previous subsection, see Figure 4. The Lagrange multiplier $\gamma$ for the coating constraint is initially equal to 0.05 , and the one for the mass constraint is initially equal to 0.1 . They are increased of $20 \%$ each five iterations. No augmented Lagrangian was used for this example.

The mass constraint is set to $40 \%$ of the whole working domain.

The algorithm is applied with two different widths of the coating, $h=0.7$ and $h=2.1$, and two different upper bounds for the geometric parameters, $m_{\max }=0.8$ and $m_{\max }=0.9$ (see Subsection 2.2.2). The results are displayed on Figure 12. One can check that the final densities feature a coating of constant width.

For the thick coating $(h=2.1)$, the shapes are smaller than for the thin coating $(h=0.7)$. Indeed, the mass of the coating is closed to the target mass, and the inside of the shape has also to be filled with a non-zero density.

The optimized structures are dehomogenized according to the dehomogenization method of Subsection 4.4. Several projected structures $\Omega_{\varepsilon}(\varphi, m)$ are displayed on Figure 13 for various values of the characteristic cell period $\varepsilon$. 

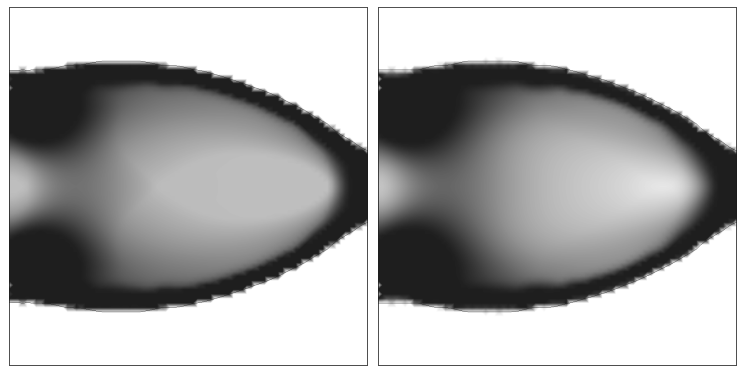

(a) $h=0.7$ and $m_{\max }=0.8$

(b) $h=0.7$ and $m_{\max }=0.9$
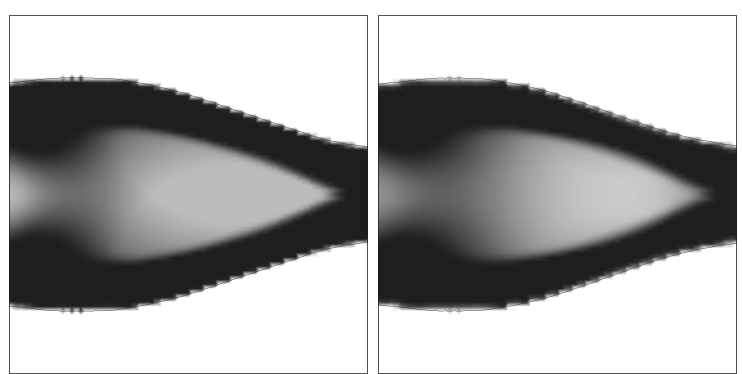

(c) $h=2.1$ and $m_{\max }=0.8\left(\right.$ d) $h=2.1$ and $m_{\max }=0.9$

Figure 12: Optimized density of the coated cantilever for two different values of coating width $h$ and of upper bound $m_{\max }$

Those genuine shapes do not require any post-treatment to clean them. Indeed, the external boundary is smooth and regular thanks to the presence of the coating.

\subsection{Optimization under design-dependent loads}

Adding a uniform pressure load of magnitude $p_{0}$ on the optimizable boundary $\Gamma$, the state equation is modified as:

$$
\left\{\begin{array}{ll}
\operatorname{div}\left(A^{*} e(u)\right)=0 & \text { in } \Omega \\
u \cdot n=0 & \text { on } \Gamma_{D} \\
A^{*} e(u) n \wedge n=0 & \text { on } \Gamma_{D} \\
A^{*} e(u) n=g & \text { on } \Gamma_{N} \\
A^{*} e(u) n=p_{0} n & \text { on } \Gamma
\end{array} .\right.
$$

Following the approach described in [6, 19], the surface integral due to the pressure load is approximated by a volume integral, as explained by (20). The compliance (3) is still minimized. Assuming that $\Gamma_{D}$ and $\Gamma_{N}$ are fixed, i.e. $\theta=0$ on these parts of the boundary, the shape derivative of the compliance is 


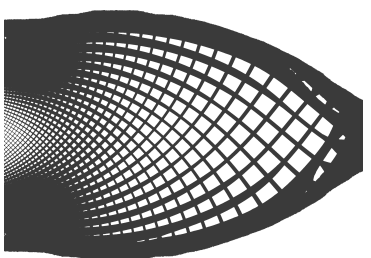

(a) $\varepsilon=0.1$

$h=0.7$ and $m_{\max }=0.8$

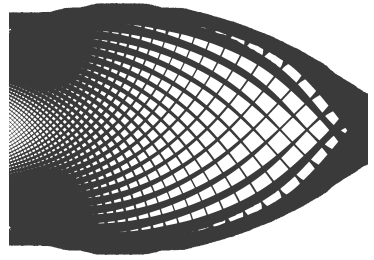

(c) $\varepsilon=0.1$

$h=0.7$ and $m_{\max }=0.9$

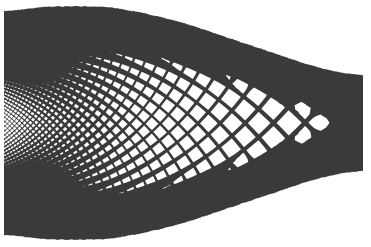

(e) $\varepsilon=0.1$

$h=2.1$ and $m_{\max }=0.8$

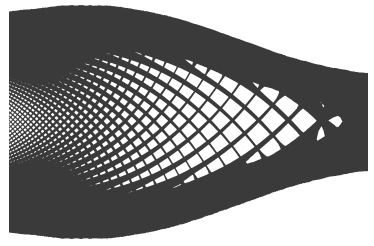

(g) $\varepsilon=0.1$

$$
h=2.1 \text { and } m_{\max }=0.9
$$

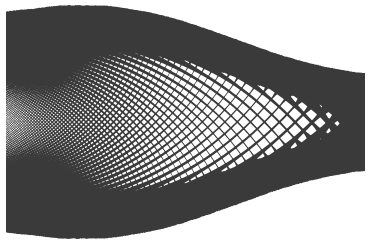

(h) $\varepsilon=0.05$

Figure 13: Projected structures $\Omega_{\varepsilon}(\varphi, m)$ for several $\varepsilon$ in the case of the coated cantilever 


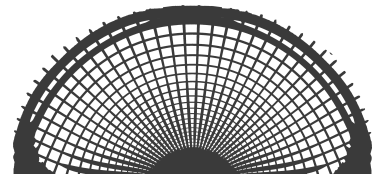

(a) $p_{0}=0$

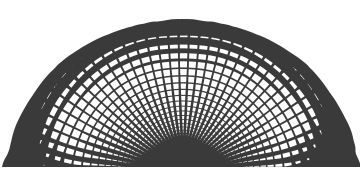

(b) $p_{0}=-0.01$

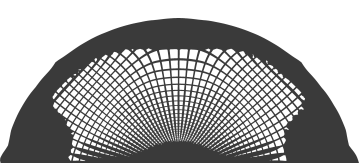

(c) $p_{0}=-0.05$

Figure 14: Dehomogenized structures $\Omega_{\varepsilon}(\varphi, m)$ under pressure loads, for several values of the pressure $p_{0}$

modified accordingly (see $[6,19])$ :

$$
\begin{aligned}
& J^{\prime}\left(\Omega, m, \alpha^{*}\right)(\theta)= \\
& -\int_{\Gamma} R\left(\alpha^{*}\right) A_{0}^{*}(m) R\left(\alpha^{*}\right)^{T} e(u): e(u) \theta . n d s \\
& +\int_{\Omega} R\left(\alpha^{*}\right) \frac{\partial A_{0}^{*}}{\partial m}(m) R\left(\alpha^{*}\right)^{T} e(u): e(u) \theta \cdot \nabla m d x \\
& +\int_{\Gamma} 2 \operatorname{div}\left(u p_{0}\right) \theta \cdot n d s .
\end{aligned}
$$

We consider almost the same bridge case, as in Figure 9. The boundary conditions are identical except the addition of the pressure load $p_{0}$ on $\Gamma$ and the sliding constraint on $\Gamma_{D}$ instead of a clamp. For this bridge test case, the boundary $\Gamma$ is just the upper boundary of the arch structure (see Figure 14). Several tests are run for different values of the pressure $p_{0}$, from 0 to -0.05 . The value 0 corresponds to the setting of Subsection 5.1.2 and it is displayed only for the sake of comparison. The second value, -0.01 , yields a pressure loading equivalent to $30 \%$ of the unit vertical load. The third value, -0.05 is taken in order to have a pressure load $50 \%$ greater than the vertical load. Notice that the precise values of the integrated pressure loads are unknown, since the length of the shape boundary is not fixed.

The target mass is equal to $30 \%$ of the mass of the working domain $D$. The upper bound on the geometric parameters is set to $m_{\max }=0.8$, and $n_{0}=3$. Genuine shapes $\Omega_{\varepsilon}(\varphi, m)$, obtained by the dehomogenisation process of Subsection 4.4, are displayed on Figure 14, for three different values of the pressure $p_{0}$, and a characteristic cell period $\varepsilon$ equal to 0.1 .

In the absence of pressure, the final structure does not feature a coating. It is quite close to the bridge structure, see Figure 11, but not exactly the same since the parameters of the algorithm are slightly different. When the magnitude of the pressure increases, a coating appears and becomes thicker. The shape optimization process takes well into account the design-dependent load.

We emphasize that such a pressure load can not be straightforwardly considered in shape optimization by the homogenization method. Indeed, an homogenized structure does not feature a clear external border. Hence, the coupled method presented here is a simple and effective way to address this problem. 


\section{Acknowledgements}

The work of G. A. and P. G.-D. is supported by the SOFIA project, funded by BPI. The work of P. G. D. was part of her PhD, funded by a CIFRE contract with SAFRAN, the support of which is kindly acknowledged. The work of G.A. is also partially supported by the project ANR-18-CE40-0013 SHAPO financed by the French Agence Nationale de la Recherche (ANR). G. A. is a member of the DEFI project at INRIA Saclay Ile-de-France.

\section{References}

[1] G. Allaire. Shape optimization by the homogenization method, volume 146. Springer Science \& Business Media, 2002.

[2] G. Allaire. Conception optimale de structures, volume 58. Springer, 2007.

[3] G. Allaire, C. Dapogny, G. Delgado, and G. Michailidis. Multi-phase structural optimization via a level set method. ESAIM: control, optimisation and calculus of variations, 20(2):576-611, 2014.

[4] G. Allaire, P. Geoffroy-Donders, and O. Pantz. Topology optimization of modulated and oriented periodic microstructures by the homogenization method. Computers \& Mathematics with Applications, 78(7):2197-2229, 2019.

[5] G. Allaire, F. Jouve, and A.-M. Toader. A level-set method for shape optimization. Comptes Rendus Mathematique, 334(12):1125-1130, 2002.

[6] G. Allaire, F. Jouve, and A.-M. Toader. Structural optimization using sensitivity analysis and a level-set method. Journal of computational physics, 194(1):363-393, 2004.

[7] G. Allaire and R. V. Kohn. Optimal design for minimum weight and compliance in plane stress using extremal microstructures. European journal of mechanics. A. Solids, 12(6):839-878, 1993.

[8] G. Allaire and O. Pantz. Structural optimization with freefem++. Structural and Multidisciplinary Optimization, 32(3):173-181, 2006.

[9] M. Bendsoe, E. Lund, N. Olhoff, and O. Sigmund. Topology optimizationbroadening the areas of application. Control and Cybernetics, 34(1):7, 2005.

[10] M. P. Bendsøe and N. Kikuchi. Generating optimal topologies in structural design using a homogenization method. Computer methods in applied mechanics and engineering, 71(2):197-224, 1988.

[11] M. P. Bendsoe and O. Sigmund. Topology optimization: theory, methods, and applications. Springer Science \& Business Media, 2003. 
[12] C. Bui, C. Dapogny, and P. Frey. An accurate anisotropic adaptation method for solving the level set advection equation. International Journal for Numerical Methods in Fluids, 70(7):899-922, 2012.

[13] M. Burger. A framework for the construction of level set methods for shape optimization and reconstruction. Interfaces Free Bound., 5(3):301$329,2003$.

[14] A. Clausen, N. Aage, and O. Sigmund. Exploiting additive manufacturing infill in topology optimization for improved buckling load. Engineering, $2(2): 250-257,2016$.

[15] C. Dapogny and P. Frey. Computation of the signed distance function to a discrete contour on adapted triangulation. Calcolo, 49(3):193-219, 2012.

[16] F. de Gournay. Velocity extension for the level-set method and multiple eigenvalues in shape optimization. SIAM J. Control Optim., 45(1):343-367, 2006.

[17] M. Delfour and J.-P. Zolésio. Shapes and geometries, volume 4 of Advances in Design and Control. Society for Industrial and Applied Mathematics (SIAM), Philadelphia, PA, 2001.

[18] M. Delfour and J.-P. Zolésio. Shape identification via metrics constructed from the oriented distance function. Control and Cybernetics, 34(1):137, 2005 .

[19] J. Desai, A. Faure, G. Michailidis, G. Parry, and R. Estevez. Topology optimization in acoustics and elasto-acoustics via a level-set method. Journal of Sound and Vibration, 420:73-103, 2018.

[20] P. D. Dunning and H. A. Kim. Introducing the sequential linear programming level-set method for topology optimization. Structural and Multidisciplinary Optimization, 51(3):631-643, 2015.

[21] P. Geoffroy-Donders, G. Allaire, and O. Pantz. 3-d topology optimization of modulated and oriented periodic microstructures by the homogenization method. Journal of Computational Physics, page to appear, 2019.

[22] J. P. Groen and O. Sigmund. Homogenization-based topology optimization for high-resolution manufacturable micro-structures. International Journal for Numerical Methods in Engineering, 113(8):1148-1163, 2018.

[23] J. P. Groen, J. Wu, and O. Sigmund. Homogenization-based stiffness optimization and projection of $2 \mathrm{~d}$ coated structures with orthotropic infill. Computer Methods in Applied Mechanics and Engineering, 349:722-742, 2019 . 
[24] L. Harzheim and G. Graf. A review of optimization of cast parts using topology optimization: I - topology optimization without manufacturing constraints. Structural and multidisciplinary optimization, 30(5):491-497, 2005 .

[25] L. Harzheim and G. Graf. A review of optimization of cast parts using topology optimization: II - topology optimization with manufacturing constraints. Structural and multidisciplinary optimization, 31(5):388-399, 2006.

[26] F. Hecht. New development in freefem++. Journal of numerical mathematics, 20(3-4):251-266, 2012.

[27] G. Michailidis. Manufacturing constraints and multi-phase shape and topology optimization via a level-set method. $\mathrm{PhD}$ thesis, Ecole Polytechnique X, 2014.

[28] J. Nocedal and S. Wright. Numerical optimization. Springer Series in Operations Research and Financial Engineering. Springer, New York, second edition, 2006.

[29] S. Osher and R. Fedkiw. Level set methods and dynamic implicit surfaces, volume 153. Springer Science \& Business Media, 2006.

[30] S. Osher and J. A. Sethian. Fronts propagating with curvature-dependent speed: algorithms based on hamilton-jacobi formulations. Journal of computational physics, 79(1):12-49, 1988.

[31] O. Pantz and K. Trabelsi. A post-treatment of the homogenization method for shape optimization. SIAM Journal on Control and Optimization, 47(3):1380-1398, 2008.

[32] P. Pedersen. On optimal orientation of orthotropic materials. Structural Optimization, 1(2):101-106, 1989.

[33] J. Podroužek, M. Marcon, K. Ninčević, and R. Wan-Wendner. Bio-inspired $3 \mathrm{~d}$ infill patterns for additive manufacturing and structural applications. Materials, 12(3):499, 2019.

[34] H. Rodrigues, J. M. Guedes, and M. Bendsoe. Hierarchical optimization of material and structure. Structural and Multidisciplinary Optimization, 24(1):1-10, 2002.

[35] J. Simon and F. Murat. Sur le contrôle par un domaine géométrique. Publication 76015 du Laboratoire d'Analyse Numérique de l'Université Paris VI, (76015):222 pages, 1976.

[36] K. Suzuki and N. Kikuchi. A homogenization method for shape and topology optimization. Computer methods in applied mechanics and engineering, 93(3):291-318, 1991. 
[37] L. Tartar. The general theory of homogenization: a personalized introduction, volume 7. Springer, 2009.

[38] M. Y. Wang, X. Wang, and D. Guo. A level set method for structural topology optimization. Computer methods in applied mechanics and engineering, 192(1-2):227-246, 2003.

[39] Y. Wang and Z. Kang. A velocity field level set method for shape and topology optimization. International Journal for Numerical Methods in Engineering, 115(11):1315-1336, 2018.

[40] J. Wu, N. Aage, R. Westermann, and O. Sigmund. Infill optimization for additive manufacturing - approaching bone-like porous structures. IEEE transactions on visualization and computer graphics, 24(2):11271140, 2018.

[41] J. Wu, W. Wang, and X. Gao. Design and optimization of conforming lattice structures. arXiv preprint arXiv:1905.02902, 2019.

[42] S. Yin, H. Chen, J. Li, T. Yu, and J. Xu. Effects of architecture level on mechanical properties of hierarchical lattice materials. International Journal of Mechanical Sciences, 157:282-292, 2019.

[43] M. Zhou, R. Fleury, Y.-K. Shyy, H. Thomas, and J. Brennan. Progress in topology optimization with manufacturing constraints. In Proceedings of the 9th AIAA MDO conference AIAA-2002-4901, 2002. 